\title{
脑空间信息学——连接脑科学与类脑人工智能的 桥梁
}

\section{骆清铭 ${ }^{1,2}$}

1. 华中科技大学生物医学工程系, 生物医学光子学教育部重点实验室, 武汉 430074;

2. 华中科技大学-武汉光电国家实验室(筹)Britton Chance生物医学光子学研究中心, 武汉 430074

E-mail: qluo@mail.hust.edu.cn

收稿日期: 2017-05-04; 接受日期: 2017-05-26; 网络版发表日期: 2017-08-17

国家自然科学基金创新研究群体项目(批准号: 61421064)和国家自然科学基金(批准号: 91232000)资助

\begin{abstract}
摘要提出脑空间信息学是示踪、测量、分析、处理和呈现跨层次多尺度脑空间信息数据的一门综合与集 成的科学. 讨论了脑空间信息学的研究内容、技术体系和关键科学问题, 分析了其学科定位, 展望了其应用前 景. 以显微光学切片断层成像为核心的全脑网络可视化技术体系的建立, 标志着脑空间信息学这一新兴交叉学 科日榛成熟. 基于具有明确时空尺度和位置信息的神经元类型、神经环路和网络、血管网络等三维精细脑结 构与功能大数据, 提取跨层次、多尺度的脑连接时空特征, 脑空间信息学将帮助科学家更好地破译脑功能与 脑疾病, 并推动类脑人工智能的发展.
\end{abstract}

关键词脑空间信息学, 全脑网络可视化, 脑连接, 数字脑, 类脑智能

从认识脑、保护脑和创造脑角度, 脑科学一般分 为神经生物学、临床神经科学和计算神经科学等学 科. 神经生物学被定义为研究人和动物的神经系统的 结构、功能及其相互关系的科学, 旨在分子、细胞、 神经回路或网络、乃至系统和整体水平上, 阐明神经 系统特别是脑的物质、能量、信息的基本活动规律, 可进一步细分为分子神经生物学、细胞神经生物学、 系统神经生物学、行为神经生物学、发育神经生物 学和比较神经生物学等分支学科.

众所周知, 脑是一个极其复杂的巨系统. 脑内神 经元、胶质细胞、血管等结构与功能信息高度复杂, 时空尺度的跨度可达十几个数量级 ${ }^{[1]}$. 脑连接及脑活
动是在不同时间-空间尺度上演化和变化的, 其物质、 能量和信息高度耦合，具有空间位置不确定性、空间 关系不确定性以及时域不确定性等. 如何在大跨度的 时间-空间尺度上研究脑连接及脑活动的演化和变化 规律是脑科学研究面临的重大挑战, 然而前述分支学 科都不能很好地定义这一新兴的交叉研究领域.

研究表明, 学科成熟的重要标志包括独立的研 究内容、成熟的研究方法和规范的学科体制等, 其 中相对成熟的研究方法或完备的技术体系至关重要. Seung ${ }^{[2]}$ 在《连接组: 造就独一无二的你》中提到, 神 经科学家缺少足够的技术去测绘神经元之间的连接, 因为这个原因, 连接主义(connectionism)从来没有被视

引用格式: 骆清铭. 脑空间信息学——连接脑科学与类脑人工智能的桥梁. 中国科学: 生命科学, 2017, 47: 1015-1024

Luo Q M. Brainsmatics — bridging the brain science and brain-inspired artificial intelligence (in Chinese). Sci Sin Vitae, 2017, 47: 1015-1024, doi: 10.1360 N052017-00094 
为一门真正的科学. 由此可见技术体系在学科发展过 程中的地位.

美国Harvard大学Lichtman教授领导的团队 ${ }^{[3]}$ 以电 子显微镜为观察手段, 用时 15 个月以 $4 \mathrm{~nm} \times 6 \mathrm{~nm} \times 28 \mathrm{~nm}$ 体素分辨率测绘了 $0.07 \mathrm{~mm}^{3} \mathrm{P} 32$ 小鼠(Mus musculus)外 侧膝状体核并重建 400 多个神经元. 美国Allen脑科学 研究所重建了 $100 \mu \mathrm{m}$ 分辨的小鼠脑连接图谱 ${ }^{[4]}$. 他们 所采用的手段都没能在小鼠全脑范围内获取单神经 元分辨水平的神经环路精细结构图谱, 当然更谈不上 获取功能连接信息. 由此可见, 如何在小鼠全脑范围 获取神经元分辨水平的结构与功能图谱在国际上仍 然是未获解决的瓶颈问题.

功能磁共振成像(functional Magnetic Resonance Imaging, fMRI)主要通过测量磁共振信号来量化血氧 饱和度和血流量的变化, 对流向特定脑区血液的变化 进行检测, 从而间接反映脑神经活动所伴随的能量消 耗. 它可同时提供脑的解剖和功能视图. 该技术的突 出优点包括无损伤、无辐射、可重复测量, 空间分辨 率在毫米量级. DNA双螺旋结构发现人之一、诺贝尔 生理学或医学奖获得者Crick和Jones ${ }^{[[]}$在给Nature的评 论中写道: “我无法忍受我们没有绘制出人类大脑的 连接图. 没有它, 很难有希望能了解我们的大脑是如 何工作的. ”他还测算过, $1 \mathrm{~mm}^{3}$ 的人脑视皮层包括超 过 40000 个神经元. 显然, fMRI离神经元分辨水平还有 很大差距.

以显微光学切片断层成像 ${ }^{[6]}$ (micro-optical sectioning tomography, MOST)为标志的全脑高分辨精准空间 定位与成像方法的日益成熟和应用, 使得在全脑范围 测量和绘制三维精细的脑连接图谱、建立标准化的 数据体系成为可能. 这里所说的三维精细脑连接图 谱不是传统的、由若干带有注释信息的、离散的断 面图片的集合, 而是指空间间隔为亚微米(准连续)、 能分辨出单个神经元、具有明确空间尺度和位置信 息的全脑结构及功能(包括基因组、转录组、蛋白质 组和代谢组等信息)连接图谱. 为此, 有必要建立规范 的脑空间信息研究科学体系, 即运用现代信息科学理 论、技术与方法, 从信息科学角度, 以高的体素分辨 率(voxel resolution), 如细胞/轴突/毛细血管分辨水平, 在全脑范围测量(surveying)与绘制(mapping)脑结构和 功能信息的三维空间分布, 如神经元投射、神经/血管
分布、基因组/蛋白组/代谢组/转录组分布等, 从而揭 示脑连接及活动的时空特征和变化规律, 如神经元和 血管走向、同类型神经元的覆盖范围、神经元之间 的时空连接特征及投射方向等. 脑空间信息学的提出 正是顺应这一发展趋势的体现.

\section{1 脑空间信息学的定义}

脑空间信息学 (brain-spatial information science, brainsmatics)是以脑连接的基本结构与功能单元为研 究对象, 揭示脑连接空间信息机制, 引导脑疾病防治 与智能技术发展的新兴交叉学科. 该学科是认知脑功 能并进而探讨意识本质的科学前沿, 是沟通脑科学与 智能科学的桥梁. 脑空间信息学将脑作为一个完整的 系统, 顺应智能技术发展需求, 以脑科学和信息科学 的基本理论为指导, 运用新兴的全脑高分辨精准空间 定位与成像方法, 同时结合多种前沿脑科学研究技术, 标记、获取、分析和可视化具有明确空间尺度和位 置的精细脑网络结构与功能信息, 从大数据中提取跨 层次、多尺度的脑连接时空特征, 揭示感知、记忆、 意识和情感等脑连接空间信息机制, 从而促进脑健康 与智能技术的跨越式发展.

\section{2 脑空间信息学的研究内容、技术体系及 关键科学问题}

这里从脑连接的示踪、测量、可视化以及时空信 息特征的提取与模拟等 4 个方面简要讨论脑空间信息 学的研究内容、技术体系及关键科学问题. 脑空间信 息学的技术体系是指贯穿脑空间信息示踪、测量、 分析、处理、呈现、传播和应用等一系列技术方法 所构成的完整技术方法的总和. 它是实现脑空间信息 从获取到应用的技术保证, 并能在自动化、时效性、 详细程度和可靠性等方面满足人们的需要.

对比脑科学的其他分支学科可以看出, 虽然研究 对象都是脑神经系统, 脑空间信息学具有相对独特而 成熟的技术体系, 特别是介观尺度的全脑高分辨精准 空间定位与成像, 为获取具有明确空间尺度和位置的 精细脑网络结构与功能信息, 并从大数据中提取跨层 次、多尺度的脑连接时空特征提供了技术保证. 与过 于理想的连接主义 ${ }^{[2]}$ 不同的是, 可行的技术体系标志 
着脑空间信息学这一新兴交叉学科日臻成熟.

\section{1 脑连接的示踪}

脑连接的示踪主要研究并运用各种示踪技术, 从 分子、突触、单细胞, 到环路、网络和脑区等不同层 次, 为多模态原位获取精细脑连接结构和功能信息, 提 供特异、高效、可控的标记方法. 19世纪末和 20 世纪 初, 意大利科学家Camillo Golgi和西班牙学者Santiago Ramón y Cajal先后开发利用高尔基银染法对神经元进 行染色, 并获得 1906年的诺贝尔生理学或医学奖, 由 此可见示踪方法的重要性.

现有示踪技术包括: 借助化学、病毒学和基因 组、蛋白质组、转录组、代谢组等组学技术, 发展 不同尺度下脑空间信息的原位标记技术, 如转基因 技术 ${ }^{[7]}$ 、示踪标记技术 ${ }^{[8]}$ (免疫标记、染色示踪、转染 标记等)、基因编辑技术 ${ }^{[9]}$ 等, 实现对不同层次脑连接 基本单元的特异、高亮标记. 从实现测量的角度, 通 常被忽视的样品制备技术 ${ }^{[10 \sim 13]}$ 往往是最重要制约因 素. 图1展示了Thy1-eYFP-H转基因小鼠脑内初级运动 皮层神经元(绿色、紫色)和初级视觉皮层神经元(黄 色、蓝色)的长程投射及分布.

Jiang等人 ${ }^{[14]}$ 研究了成年小鼠新皮层不同神经元 的形态差异并初步证实神经元可被归类为有限的细 胞类型, 且每一种神经元类型都有其固定的连接模式. 据此, 可以大胆假设, 不同类型的神经元、神经环路/ 网络与神经系统之间的关系, 可以类比于化学元素、
化合物与生命有机体, 也可类比于分立的电子元器件 (如电阻、电容、电感、三极管等)、基础电路(如电 源电路、音频电路、放大电路等)与电子系统(通信电 子系统、飞机电子系统、导弹电子系统等).

关键科学问题主要包括: (i) 如何增强脑空间信 息的示踪效率, 实现对微观精细结构、介观完整形态 和宏观整体信息的高对比度、完整标记? (ii) 如何提 高标记的特异性, 实现不同类型神经元和脑细胞(可进 一步区分为亚类)、神经环路、以及由多个环路所组 成的神经网络乃至神经系统等不同层次结构和功能 信息的示踪? 如何实现针对特定类型神经元、环路和 网络的稀疏数量可控、跨突触级数可控?

\section{2 全脑范围脑连接的测量}

全脑范围脑连接的测量主要研究不同尺度下全 脑、高分辨精准成像与空间定位方法, 建立与之相应 的定位基准, 并将数据采集过程标准化, 实现具有明确 空间尺度和定位含义的精细脑网络结构与功能信息 的获取和分析, 为提取脑连接时空特征提供跨层次、 多尺度的大数据, 从而揭示和掌握脑空间信息的时空 变化特征及规律.

在介观尺度下最有特色的全脑范围脑连接的测量 技术是以MOST为基础的系列全脑高分辨精准空间定 位与成像技术 ${ }^{[15 ~ 22]}$. 此外, 连续双光子层析成像 ${ }^{[23]}$ (serial two-photon tomography, STP)、光片照明显微成像 ${ }^{[24]}$
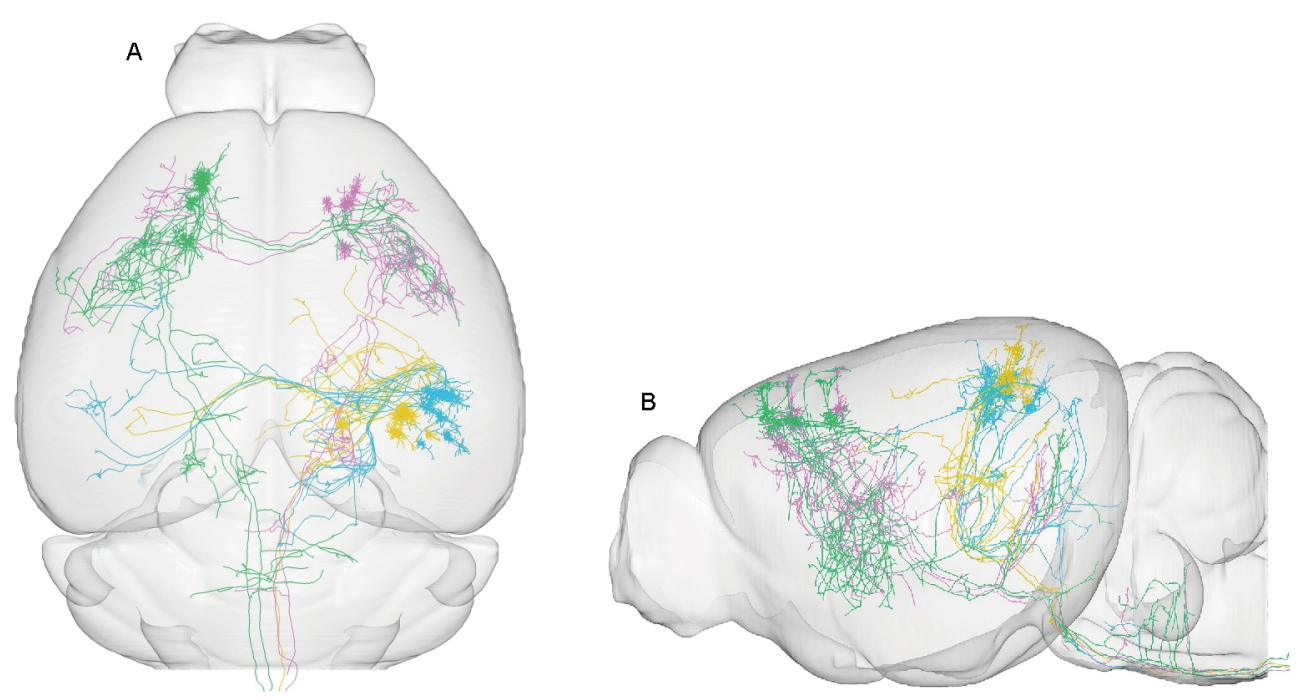

图 1 小鼠初级运动皮层神经元(绿色、紫色)和初级视觉皮层神经元(黄色、蓝色)的长程投射及分布 
(light sheet microscopy, LSM)、多光子显微成像 ${ }^{[25]}$ 等也可以为研究脑空间连接信息提供部分技术支 持. 介观尺度的测量技术可以与宏观尺度的测量 技术, 如 $\mathrm{fMRI}^{[26]}$ 、正电子发射断层成像 ${ }^{[27]}$ (positron emission tomography, PET)、脑电 ${ }^{[28]}$ (electroencephalogram, EEG)、功能近红外光学成像 ${ }^{[29-31]}$ (functional near-infrared imaging, fNIRI)、X射线计算机层析成像 (X-CT)、超声成像等, 以及微观尺度的成像技术, 如 电子显微镜成像 ${ }^{[32]}$ 、超分辨荧光纳米显微成像 ${ }^{[33]}$ 等, 相互配合与交互. 脑空间信息测量的定位基准和数 据采集过程的标准化是推动脑科学研究成果产业化 的重要保障, 这也是脑空间信息测量技术最迫切需 要解决的问题.

关键科学问题主要包括: (i) 如何实现介观和微 观尺度下脑连接空间信息的获取? 现有技术已能解析 宏观尺度下的脑连接, 但仍缺乏介观和微观空间尺度 水平时空变化的测量手段. (ii) 如何建立不同尺度的 定位基准? 定位基准是在不同条件下所获取的脑空间 信息进行匹配及比较的前提条件. 目前, 宏观尺度的 定位基准已初步建立, 尚缺乏介观及微观尺度的定位 基准, 亟待确定. (iii) 如何实现测量标准化? 测量设备 及测量过程的全过程标准化管理是保证脑空间信息 数据可靠性和有效性的重要前提, 也是开展对比性研 究的重要基础.

\section{3 脑连接的可视化}

研究内容主要包括脑空间信息的数据可视化、科 学可视化、信息可视化和知识可视化 ${ }^{[34-36]}$. 可视化的 目标是将跨层次、多维度的脑空间信息时空变化过 程, 通过运用高维数据场、虚拟现实等技术直观、高 效地呈现出来, 以供用户观察、模拟和计算. 开展脑 连接的可视化研究一方面需要建立涵盖数据管理、 运算、分析和展现等手段在内的一整套技术方案, 另 一方面还要构建数据编码标准、数据表示标准、数 据质量标准、数据交换标准、空间定位标准、流程 控制规范等完整标准体系. 实现脑连接的可视化首先 要完成脑空间信息大数据的数字化和知识化, 其中, 数字化又是知识化的基础, 研究从多维图像大数据中 准确、高效地分割重建出脑区核团、神经元形态等 特定结构, 实现图像到图形的变换. 知识化是研究运 用计算机辅助技术并结合专家知识, 将数字化的图形
图像数据准确变换为结构化的知识, 并通过数据库、 图谱等基本组织形式对脑空间信息知识进行收集、 分类、检索和调用.

三维脑连接信息的可视化对于建立脑认知模型 并最终理解脑工作机制至关重要. 16世纪意大利艺术 家Giuseppe Arcimboldo 曾神奇地把蔬菜、水果和花组 合成人像, 犹如几十种不同的化学元素最终能组成人 体. 人类依据还原论思想, 成功地对自然界加深了认 识, 然而另一方面, 也需要整体论或系统论思想的指 导. 如何实现对海量脑连接大数据的准确展示, 将是 脑科学研究面临的重大挑战.

脑空间信息数据量巨大, 技术体系涉及大数据存 储与传输, 在数据结构、数据压缩、云存储等多方面 都需要技术支撑 $\left.{ }^{[37} 41\right]$. 此外, 在数字化方面, 需要运用 和发展现代数字图像处理、云计算、统计学、机器 学习等技术; 在知识化方面, 需要借鉴和采用数据挖 掘、数据库、情报学等技术; 在可视化方面, 则需要 运用和发展计算机图形学、虚拟现实等技术.

关键科学问题主要包括: (i) 如何灵活运用专家 介入和自动化技术, 在复杂大数据中实现脑空间信息 准确而高效的数字化? (ii) 如何应用虚拟现实等技 术, 对时空变化过程中获取的大数据进行交互式可视 化? (iii) 如何利用有限的计算资源, 实现对大数据的 存储、传输、运算和呈现?

\section{4 脑连接时空信息特征的提取与模拟}

主要内容是通过整合多模态、跨层次、多尺度 的全脑高时空分辨信息, 模拟特定功能(运动、视觉、 听觉、情感、记忆等)神经环路和网络的时空动态过 程, 进行空间上的形态分析、时间上的过程分析和发 生上的因果分析, 实现对脑空间信息的定性解译和定 量反演, 揭示脑连接的时空变化规律, 进而实现全脑 的功能模拟, 为类脑和智能技术提供全新的理论基础.

采用全脑网络可视化(visible brain-wide networks, $\mathrm{VBN})$ 技术测绘的小鼠桶状脑皮层发现 ${ }^{[22]}$, 同一脑区存 在多种形态的神经元, 其投射模式也不一样, 有局部, 也有长程. 如图2(A)所示, 总线有限的数据传输速率 被称为“冯·诺依曼瓶颈”, 显然, 脑信息处理的机制是 完全不一样的, 但实际情况是什么? 不同脑区之间如 何实现信息交互? 是图2(B)所示的具有少量捷径连接 的小世界网络 ${ }^{[2]}$ 吗? 需要在全脑范围、运用系统生物 


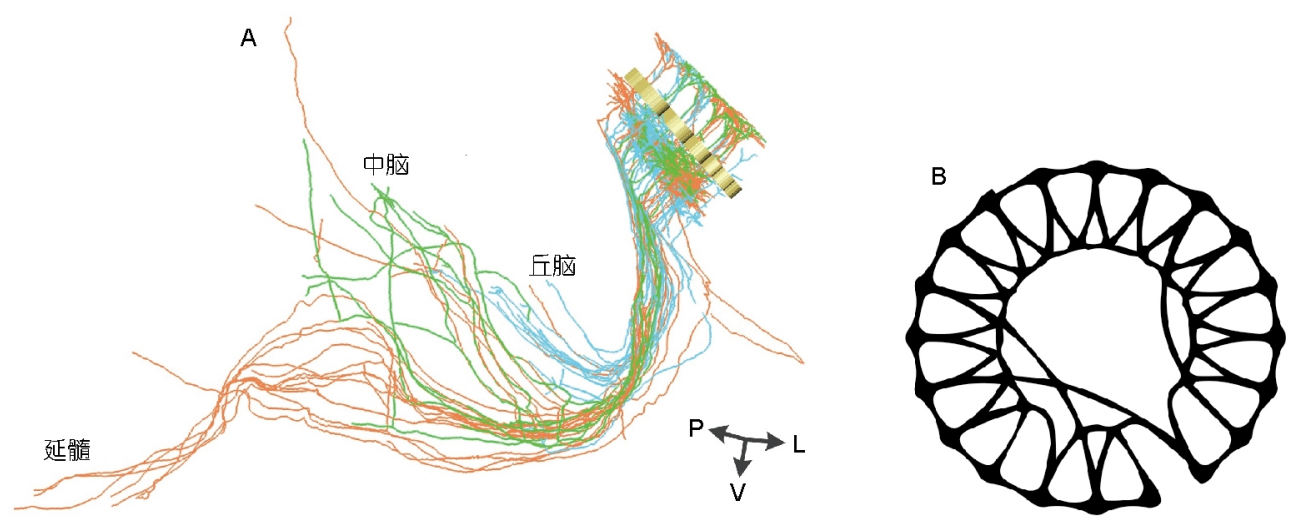

图 2 脑网络连接与小世界网络的对比

$\mathrm{A}$ : 小鼠桶状皮层同一脑区存在多种形态及多种投射路径的神经元实测结果 ${ }^{[22]}$; B: 具有少量捷径连接的小世界网络 ${ }^{[2]}$

学思想来研究这个问题, 通过建立跨层次多尺度模型, 模拟并最终认识脑连接的基本规律.

技术体系中, 需要采用对所获得的数据进行解 析和理解的方法, 包括统计信号处理 ${ }^{[43]}$ 、高维数据 分析 ${ }^{[44]}$ 、大数据处理 ${ }^{[45]}$ 等, 而对所理解的知识进行建 模的方法, 则包括统计建模 ${ }^{[46]}$ 、机器学习 ${ }^{[47]}$ 、深度学 习[48]等.

关键科学问题主要包括: (i) 如何从多模态跨层 次多尺度和多维度的脑连接大数据中, 揭示特定脑功 能的时空变化规律? (ii) 如何针对类脑研究的需求, 构建脑空间信息的数学模型?

\section{3 大数据时代的脑空间信息学}

大脑非常复杂, 全面理解大脑必将带来多层次、 多模式的海量数据. 例如, 基于 MOST成像技术, 以微 米体素分辨率采集一个完整小鼠脑的数据量达 $8 \mathrm{~TB}^{[6]}$, 如果是苂光图像数据量则更大 ${ }^{[22]}$. 对于人脑而言, 若 以相同体素分辨率采集, 预计数据量将达到 $11 \mathrm{~PB}$, 相 当于 20 万部 $4 \mathrm{~K}$ 高清电影, 或 500 家三甲医院产出的全 年数据. 如果用 $10 \mathrm{M}$ 宽带, 仅数据传输就需要耗时 300 年. 此外, 脑研究中数据类型具有多样性, 维度、深 度、密度、数据结构、压缩方式, 以及所代表的物理 量及其对应的生物信息量各不相同. 因此, 脑研究产 生的海量数据必将对数据处理、存储、分析、管理 和共享等方式带来全方位的挑战.

数据的计算、存储、传输等技术的需求从某种意 义上来讲具有“大数据”的共性, 但如何科学地组织、
共享和管理这些神经信息数据, 提高数据的使用率和 价值, 是对现代信息技术特别是计算机技术提出了前 所未有的巨大挑战 ${ }^{[49]}$. 大数据的计算离不开高性能计 算领域的参与, 欧盟“人类脑计划”在巨型计算机上对 人脑建模 ${ }^{[50]}$, 通过超级计算机描绘和模拟大脑神经元 活动的海量数据, 对大脑的功能和活动进行模拟. 美 国“脑计划”也将建立大规模神经信息数据计算平台 列为重要任务之一 ${ }^{[51]}$.

大数据时代, 开展脑空间信息学研究正当时, 机 遇与挑战并存.

\section{4 脑空间信息学的应用}

具有跨层次、多尺度时空变化特征的脑空间信 息学有望在绘制脑结构及功能的三维甚至四维图谱, 并进而在认知脑功能、防治脑疾病和发展智能技术 等方面发挥重要作用.

\section{1 认知脑功能}

利用所建立的脑空间信息知识库, 回答神经元种 类及绘制脑图谱等问题, 并对研究认知和行为的神经 活动机制等脑科学问题提供基础数据. 基于脑内原位 多种信息共存的空间信息构建的模型将为脑科学研 究提供模拟和分析平台.

由于在介观尺度缺乏有效的研究工具, 目前对高 等哺乳动物神经环路连接信息的认识还极度贵之. 复 杂脑功能往往需要局部神经环路和长程神经环路的 协同作用才能完成, 其中长程神经环路可能从皮层一 
直投射至脊髓, 几乎跨越全脑范围 ${ }^{[15,22]}$. 小鼠全脑在任 意方向上都是厘米量级; 脑中的特定结构, 如海马, 在 毫米量级; 一般神经元胞体的直径约为 $5 \sim 30 \mu \mathrm{m}$, 胞体 发出的突起直径往往小于 $1 \mu \mathrm{m}$. 要在全脑范围获取精 细神经结构, 需要跨越 $4 \sim 5$ 个数量级的几何尺度进行 高分辨率成像——这是现有成像技术必须克服的技 术瓶颈. 目前, 人们仅对拥有 302 个神经元的线虫进行 了较完整的神经环路研究 ${ }^{[52]}$, 而对于拥有约 10 万个神 经元的果蝇(Drosophila melanogaster)脑的相关研究还 只是刚刚起步 ${ }^{[53]}$. 相比之下, 高等哺乳动物大脑由数 亿到数百亿计的神经元构成, 在介观尺度绘制哺乳动 物脑连接图谱对传统成像技术而言无疑是不可能完 成的任务. 作为脑空间信息学领域最有特色的研究手 段之一, 以MOST为基础的全脑高分辨精准空间定位 与成像技术体系的日益成熟, 为在介观尺度 (具有神经 元/细胞分辨度)建立小鼠或非人灵长类(狝猴) 全脑网 络精细结构与功能连接图谱提供了重要的研究工具 和技术平台.

\section{2 防治脑疾病}

通过全脑空间精细信息的整合, 对比不同模态下 脑部结构与功能的差异, 有助于系统地研究脑部疾病 及大脑发育的机制, 为脑部重大疾病如神经遗传与发 育性疾病(自闭症等)、精神性疾病(抑郁症等)和神经 退行性疾病 (老年痴呆症等)的临床防治提供支撑, 并 为儿童教育等提供理论指导.

脑疾病防治是脑科学研究最重要的需求导向之 一. 由于对脑的结构和功能缺乏认识, 不能及时有效 地治疗老年痴呆、帕金森氏病、抑郁和自闭等脑疾 病, 导致脑疾病已经成为社会负担最重的病种 ${ }^{\left[{ }^{[4]}\right.}$. 在 单神经元分辨水平揭示全脑神经环路的空间连接信 息, 不仅是阐明各种高级脑功能机制所必需的, 也是 彻底认识和征服脑疾病的重要前提. 特定神经系统疾 病往往伴随着相关神经环路的异常. 获得病理模型下 特定神经环路精细图谱, 对研发脑重大疾病的早期诊 断、精准干预(包括病前干预和疗后康复)的新技术、 新方法是至关重要的.

理想情况下, 完美模拟大脑不仅意味着知晓了大 脑如何工作, 也将知道脑功能被破坏的原因, 即脑疾 病的致病机制. 控制论的创始人Wiener ${ }^{[55]}$ 就鼓励人们 从计算机工作原理的角度去理解人类大脑. 通过有效
的计算机模拟, 有望使科学家能够厘清脑部疾病的某 些细节, 进而篮选出可行的治疗途径, 甚至革命性地 改观现有的药物研发方式, 从而形成重要的应用价值.

\section{3 发展类脑智能技术}

通过模拟、解译和反演等多种手段, 利用脑空间 信息模型探究记忆、学习、决策等原理,构建并规 范全脑空间的功能计算模型, 在类脑人工智能的设计 上模拟仿真和再现脑局部和整体功能的工作结构, 发 展类脑人工智能算法, 以此让机器获得更好的概括能 力、更好的抽象能力以及对稀疏数据的使用能力.

经过 60 年的发展, 人工智能已经取得了一系列令 人瞩目的成就. 1997 年, IBM深蓝计算机因为击败了 世界国际象棋冠军卡斯帕罗夫而名声大噪. 2016年, Google的AlphaGo又在全世界人民面前大败围棋世界 冠军李世石, 掀起了智能技术新一轮的热潮. 智能技 术如今已成功应用在非常多的领域, 例如, 机器翻译、 智能控制、专家系统、机器人学、语言和图像理解、 航天应用等重要领域, 以代替人类去执行复杂或规模 庞大的任务. 但是, 现有的智能技术还限制于弱人工 智能的发展阶段, 它的显著特征是仅具备某项认知能 力, 无法推广至实现其他功能. 谭铁牛院士形象地评 价“有智能, 没有智慧; 有智商, 没有情商; 会计算, 不会 算计; 有专能, 无全能”. 究其原因, 还是人工智能向脑 工作机制的学习还不够, “类脑”不够, 当然人类对脑的 认识本身也还十分粗浅. 目前的人工神经网络只是参 考了神经元间的部分拓扑结构而搭建的数学模型, 其 核心算法是计算机科学研究者发明的,而非来自于生 物神经系统的解析, 尚与大脑的原理和能力相去甚远.

瑞士洛桑联邦理工学院的“蓝脑计划”,[56]从 2005 年 开始就试图模拟人脑功能, 其初始目标是对构成鼠脑 新皮层中功能柱单元的 1 万个神经元及 3 千万个突触 连结进行模拟. 这个模型虽然基于十几年来的各种实 验数据, 如神经形态学、基因表达、离子通道、突触 连接, 以及很多鼠脑活动的电生理记录, 但仍然不是 真实完整的神经元网络. 美国脑计划于在2015年启动 了脑皮层模拟项目 (MICrONS) $)^{[57]}$, 旨在通过重建一立 方毫米脑区内的神经环路连接, 模拟脑皮层功能, 研 发下一代机器智能系统. 酝酿中的中国脑计划也将类 脑研究列为重要目标之一.

由此可见, 脑空间信息机制研究是认知脑功能并 
发展类脑人工智能的必由之路. 智能技术正从狭义的 人工智能向类脑人工智能发展, 基于脑连接三维甚至 四维图谱的脑空间信息学的发展将是成败的关键.

综上, 有理由认为, 脑空间信息学研究将为理解 脑功能与防治脑疾病打下坚实基础, 也为发展类脑人 工智能技术提供重要指导. 中国脑计划已箭在弦上, 以重大需求为导向, 通过发展核心关键技术, 建立科 学的研究体系, 应该成为中国脑计划实现突破并引领 国际的重要路径 ${ }^{[58-60]}$.

\section{5 讨论与展望}

考察生物信息学的发展历程我们可以看到, 正是 由于DNA测序技术的快速发展, 催生了一个从积累数 据向解释数据的时代转变, 这是历史发展的必然. 同 样, 随着介观尺度全脑网络可视化 ${ }^{[20,21]}$ 技术的发展, 产 生了 PB级脑空间信息的数据. 如何利用信息技术, 特 别是计算机技术, 解析脑连接时空变化特性, 正成为 脑科学研究的前沿. 因此, 脑空间信息学是脑科学与 信息科学交叉融合与发展的必然结果.

从研究方法角度, 以VBN为核心技术体系的脑空 间信息学是系统论研究和还原论研究的重要结合点.

脑空间信息学是由脑科学和信息科学高度交叉 而形成的一门新兴的学科, 其最重要的目标是认识脑, 并且其研究成果对于保护脑和创造脑同样意义重大.
脑空间信息学最重要的特点是采用了以VBN为核心 的独特技术体系, 这一新的技术体系以亚微米甚至更 高的体素分辨率, 全局(全脑范围)、动态(脑发育的不 同阶段)地为脑科学研究提供前所未有的数据, 包括 神经元分辨水平的基因组、转录组、蛋白质组和代 谢组等表达信息. 脑空间信息学不仅在研究对象上面 临着人类的终极挑战一一脑, 在研究手段方面, 对现 代信息科学与技术的发展也提出了前所未有的挑战. 为此, 有必要对这一新兴学科进行更深入更全面的规 划和建设, 不断完善其学科体系, 引领脑科学与智能 技术的发展.

需要特别强调的是, 支撑脑空间信息学的技术体系 不只是VBN, 也包括以电子显微镜为代表的局域脑组 织高分辨成像和以 $\mathrm{fMRI}$ 为代表的宏观水平的全脑成像. 电子显微镜的成像分辨率很高, 但成像范围极其有限, Luo等人 ${ }^{[6]]}$ 测算过, $1 \mathrm{~mm}^{3}$ 的脑组织, 如果用电子显微镜 成像, 需要 1 万人/年的工作量. fMRI虽然能快速观测全 脑, 但不能在神经元/细胞分辨水平真实反映脑结构与 功能活动 ${ }^{\left[{ }^{[}\right]}$. 只有基于MOST或荧光MOST(fluorescence micro-optical sectioning tomography, fMOST)的技术体 系既能在亚细胞分辨水平、又能在全脑范围实现脑结 构与功能的观测. MOST/fMOST与电子显微镜和fMRI 等技术互相补充、融合, 将构建出更完备的脑空间信 息技术体系框架, 使得脑空间信息学不再是空中楼阁.

致谢中国科学院武汉物理与数学研究所叶朝辉、国家自然科学基金委员会曹河圻、华中科技大学枈辉提出了 重要修改意见, 华中科技大学李安安、袁菁、李向宁、许形辉、杨孝全、张智红、王平、黄松林、张小宇、吴具、 齐毅松、周伟、曾绍群、施华等参加了调研与讨论.

\section{参考文献}

1 Mitra P P. The circuit architecture of whole brains at the mesoscopic scale. Neuron, 2014, 83: 1273-1283

2 Seung S. Connectome: how the brain's wiring makes us and who we are. Houghton Mifflin Harcour, 2013, 117: 1513-1514

3 Morgan J L, Berger D R, Wetzel A W, et al. The fuzzy logic of network connectivity in mouse visual thalamus. Cell, 2016, 165: 192-206

4 Oh S W, Harris J A, Ng L, et al. A mesoscale connectome of the mouse brain. Nature, 2014, 508: 207-214

5 Crick F, Jones E. Backwardness of human neuroanatomy. Nature, 1993, 361: 109-110

6 Li A, Gong H, Zhang B, et al. Micro-optical sectioning tomography to obtain a high-resolution atlas of the mouse brain. Science, 2010, 330: 1404-1408

7 Huang Z J, Zeng H. Genetic approaches to neural circuits in the mouse. Annu Rev Neurosci, 2013, 36: 183-215

8 Marshel J H, Mori T, Nielsen K J, et. al. Targeting single neuronal networks for gene expression and cell labeling in vivo. Neuron, 2010, 67: $562-574$

9 Izpisua Belmonte J C, Callaway E M, Caddick S J, et al. Brains, genes, and primates. Neuron, 2015, 86: 617-631 
10 Zhang B, Li A, Yang Z, et al. Modified Golgi-Cox method for micrometer scale sectioning of the whole mouse brain. J Neurosci Methods, 2011, 197: $1-5$

11 Wang Q, Li A, Gong H, et al. Quantitative study on the hygroscopic expansion of spurr resin to obtain a high-resolution atlas of the mouse brain. Exp Biol Med (Maywood), 2012, 237: 1134-1141

12 Yang Z, Hu B, Zhang Y, et al. Development of a plastic embedding method for large-volume and fluorescent-protein-expressing tissues. PLoS ONE, 2013, 8: e60877

13 Xiong $\mathrm{H}$, Zhou Z, Zhu M, et al., Chemical reactivation of quenched fluorescent protein molecules enables resin-embedded fluorescence microimaging. Nat Commun, 2014, 5: 3992

14 Jiang X, Shen S, Cadwell C R, et al. Principles of connectivity among morphologically defined cell types in adult neocortex. Science, 2015, 350: 6264: aac9462

15 Gong H, Zeng S, Yan C, et al. Continuously tracing brain-wide long-distance axonal projections in mice at a one-micron voxel resolution. NeuroImage, 2013, 74: 87-98

16 Xu D, Jiang T, Li A, et al. Fast optical sectioning obtained by structured illumination microscopy using a digital mirror device. J Biomed Opt, 2013, 18: 060503

17 Zheng T, Yang Z, Li A, et al. Visualization of brain circuits using two-photon fluorescence micro-optical sectioning tomography. Opt Express, 2013, 21: 9839-9850

18 Qi X L, Xiong H Q, Lv X H, et al. Improved detectability of neuronal connectivity on mechanical sectioning setup by using confocal detection. J Biomed Opt, 2013, 18: 50506

$19 \mathrm{Wu}$ J, He Y, Yang Z, et al. 3D BrainCV: simultaneous visualization and analysis of cells and capillaries in a whole mouse brain with one-micron voxel resolution. NeuroImage, 2014, 87: 199-208

20 Luo Q M. Visible brain-wide networks at single-neuron resolution with micro-optical sectioning tomography. Conf Laser Electr, 2014

21 Yuan J, Gong H, Li A, et al. Visible rodent brain-wide networks at single-neuron resolution. Front Neuroanat, 2015, 9: 70

22 Gong H, Xu D, Yuan J, et al. High-throughput dual-colour precision imaging for brain-wide connectome with cytoarchitectonic landmarks at the cellular level. Nat Commun, 2016, 7: 12142

23 Ragan T, Kadiri L R, Venkataraju K U, et al. Serial two-photon tomography for automated ex vivo mouse brain imaging. Nat Meth, 2012, 9: $255-258$

24 Dodt H U, Leischner U, Schierloh A, et al. Ultramicroscopy: three-dimensional visualization of neuronal networks in the whole mouse brain. Nat Meth, 2007, 4: 331-336

25 Ouzounov D G, Wang T, Wang M, et al. In vivo three-photon imaging of activity of GCaMP6-labeled neurons deep in intact mouse brain. Nat Meth, 2017, 14: 388-390

26 Eisenberger N I, Lieberman M D, Williams K D. Does rejection hurt? An fMRI study of social exclusion. Science, 2003, 302: 290-292

27 Duncan J, Seitz R J, Kolodny J, et al. A neural basis for general intelligence. Science, 2000, 289: 457-460

28 Makeig S, Westerfield M, Jung T P, et al. Dynamic brain sources of visual evoked responses. Science, 2002, 295: 690-694

29 Sun B, Zhang L, Gong H, et al. Detection of optical neuronal signals in the visual cortex using continuous wave near-infrared spectroscopy. NeuroImage, 2014, 87: 190-198

30 Sun J Y, Sun B L, Zhang L, et al. Correlation between hemodynamic and electrophysiological signals dissociates neural correlates of conflict detection and resolution in a Stroop task: a simultaneous near-infrared spectroscopy and event-related potential study. J Biomed Opt, 2013, 18: 6014

31 Zhang Z, Sun B, Gong H, et al. A fast neuronal signal-sensitive continuous-wave near-infrared imaging system. Rev Sci Instruments, 2012, 83: 094301-094301

32 Kasthuri N, Hayworth K J, Berger D R, et al. Saturated reconstruction of a volume of neocortex. Cell, 2015, 162: 648-661

33 Balzarotti F, Eilers Y, Gwosch K C, et al. Nanometer resolution imaging and tracking of fluorescent molecules with minimal photon fluxes. Science, 2017, 355: 606-612

34 Akil H, Martone M E, Van Essen D C. Challenges and opportunities in mining neuroscience data. Science, 2011, 331: 708-712

35 Walter T, Shattuck D W, Baldock R, et al. Visualization of image data from cells to organisms. Nat Methods, 20107, 3: S26-S41

36 Chen M, Ebert D, Hagen H, et al. Data, information, and knowledge in visualization. IEEE Computer Graph Appl, 2009, 29: 12-19

37 Quan T, Zheng T, Yang Z, et al. NeuroGPS: automated localization of neurons for brain circuits using L1 minimization model. Sci Rep, 2013, 3: 1414

38 Yan C, Li A, Zhang B, et al. Automated and accurate detection of soma location and surface morphology in large-scale 3D neuron images. PLoS 
ONE, 2013, 8: e62579

39 Ding W, Li A, Wu J, et al. Automatic macroscopic density artefact removal in a Nissl-stained microscopic atlas of whole mouse brain. J Microscopy, 2013, 251: 168-177

40 Quan T, Zhou H, Li J, et al. NeuroGPS-Tree: automatic reconstruction of large-scale neuronal populations with dense neuritis. Nat Methods, 2016, 13: 51-54

41 Feng Z, Li A, Gong H, et al. An automatic method for nucleus boundary segmentation based on a closed cubic spline. Front Neuroinform, 2016, 10: 21

Whigham P A, Dick G, Parry M. Collective dynamics of 'small-world' networks. Nature, 1998, 393: 440-442

Oweiss K G. Statistical Signal Processing for Neuroscience and Neurotechnology. Burlington: Academic Press/Elsevier, 2010

44 Duchesne S, Valdivia F, Mouiha A, et al. Single time point high-dimensional morphometry in Alzheimer's disease: group statistics on longitudinally acquired data. Neurobiol Aging, 2015, 36 Suppl 1: S11-S22

45 Fan J, Han F, Liu H. Challenges of Big Data analysis. Natl Sci Rev, 2014, 1: 293-314

46 Srivastava A, Lee A B, Simoncelli E P, et al. On advances in statistical modeling of natural images. J Math Imag Vision, 2003, 18: 17-33

47 Pereira F, Mitchell T, Botvinick M. Machine learning classifiers and fMRI: a tutorial overview. NeuroImage, 2009, 45: S199-S209

48 LeCun Y, Bengio Y, Hinton G. Deep learning. Nature, 2015, 521: 436-444

49 Landhuis E. Neuroscience: big brain, big data. Nature, 2017, 541: 559-561

50 Markram H, Muller E, Ramaswamy S, et al. Reconstruction and simulation of neocortical microcircuitry. Cell, 2015, 163: 456-492

51 Bargmann C, Newsome W, Anderson A, et al. BRAIN 2025: a scientific vision. Brain Research Through Advancing Innovative Neurotechnologies (BRAIN) Working Group Report to the Advisory Committee to the Director, NIH. Available online at: http://www.nih.gov/science/brain/2025/ (US National Institutes of Health, 2014), 2014

52 Long F, Peng H, Liu X, et al. A 3D digital atlas of C. elegans and its application to single-cell analyses. Nat Methods, 2009, 6: 667-672

53 Chiang A S, Lin C Y, Chuang C C, et al. Three-dimensional reconstruction of brain-wide wiring networks in Drosophila at single-cell resolution. Curr Biol, 2011, 21: 1-11

54 Whiteford H A, Ferrari A J, Degenhardt L, et al. The global burden of mental, neurological and substance use disorders: an analysis from the Global Burden of Disease Study 2010. PLoS ONE, 2015, 10: e0116820

55 Wiener N. Cybernetics or, Control and Communication in the Animal and the Machine, 2d ed., New York: M.I.T. Press, 1961

56 Markram H. The blue brain project, nature reviews. Neuroscience, 2006, 7: 153-160

57 Underwood E. Neuroscience. Barcoding the brain. Science, 2016, 351: 799-800

58 杨雄里. 为中国脑计划呐喊. 中国科学: 生命科学, 2016, 46: 201-202

59 郭爱克. 智能时代脑科学的核心是探索智力的本质及其实现. 中国科学: 生命科学, 2016, 46: 203-205

60 骆清铭. 需求导向, 基础领先, 技术取胜. 中国科学: 生命科学, 2016, 46: 206-207

61 Luo L, Callaway E M, Svoboda K. Genetic dissection of neural circuits. Neuron, 2008, 57: 634-660 


\title{
Brainsmatics-bridging the brain science and brain-inspired artificial intelligence
}

\author{
LUO QingMing ${ }^{1,2}$ \\ 1 Key Laboratory for Biomedical Photonics of Ministry of Education, Department of Biomedical \\ Engineering, Huazhong University of Science and Technology, Wuhan 430074, China; \\ 2 Britton Chance Center for Biomedical Photonics, Huazhong University of Science and Technology-Wuhan \\ National Laboratory for Optoelectronics, Wuhan 430074, China
}

We propose a new approach of brain-spatial information science, abbreviated to brainsmatics, which refers to the integrated, systematic approach of tracing, measuring, analyzing, managing and displaying cross-level brain spatial data with multi-scale resolution. We discussed its research contents, technological systems and key scientific problems, analyzed its discipline orientation, and forecasted the applications. Taking the micro-optical sectioning tomography (MOST) serial techniques as the core, we have developed a multidisciplinary complete technical system of visible brain-wide network (VBN), which makes brainsmatics more mature. Based on big data of three-dimensional fine structural and functional imaging of neuron types, neural circuits and networks, vascular network et al, with definite temporal-spatial resolution and specific spatial locations, brainsmatics makes it possible to better decipher the brain function and disease and promote the brain-inspired artificial intelligence by extracting cross-level and multi-scale temporal-spatial characteristics of brain connectivity.

brainsmatics, visible brain-wide network, brain connectivity, digital brain, brain-inspired artificial intelligence

doi: $10.1360 / \mathrm{N} 052017-00094$ 